TITLE:

\title{
Observation of rovibrational dephasing of molecules in parahydrogen crystals by frequency domain spectroscopy
}

\author{
AUTHOR(S): \\ Katsuki, H; Momose, T
}

\section{CITATION:}

Katsuki, H ... [et al]. Observation of rovibrational dephasing of molecules in parahydrogen crystals by frequency domain spectroscopy. PHYSICAL REVIEW LETTERS 2000, 84(15): 3286-3289

ISSUE DATE:

2000-04-10

URL:

http://hdl.handle.net/2433/50040

RIGHT:

Copyright 2000 American Physical Society 


\title{
Observation of Rovibrational Dephasing of Molecules in Parahydrogen Crystals by Frequency Domain Spectroscopy
}

\author{
Hiroyuki Katsuki and Takamasa Momose* \\ Department of Chemistry, Graduate School of Science, Kyoto University, Kyoto 606-8502, Japan \\ and Japan Science and Technology Corporation (JST), Kyoto 606-8502, Japan
}

(Received 1 December 1999)

\begin{abstract}
Rotation-vibration transitions of methane molecules embedded in parahydrogen crystals were investigated through Fourier transform infrared spectroscopy. Each transition shows extremely sharp peaks with a Lorentzian line shape profile, which indicates the spectra are free from inhomogeneous broadening. The steep temperature dependence of the linewidths observed in the range between 3.7 and $8.5 \mathrm{~K}$ is interpreted to be a result of the pure dephasing relaxation mechanism. A remarkable difference in population relaxation widths between stretching and bending vibrational excited states was also found.
\end{abstract}

PACS numbers: 33.20.Ea, 67.80.-s

Vibrational relaxation of molecules in solids has been the subject of a number of experimental [1-6] and theoretical $[7,8]$ studies over the last 30 years because it gives us useful information on the interaction of an impurity molecule with its surroundings. From a spectroscopic point of view, the vibrational relaxation is obtained by both time domain and frequency domain spectroscopies. The dephasing time, $T_{2}$, obtained by time resolved experiments is related to the full width at half of the maximum (FWHM) of the transition, $\gamma$, observed by the frequency domain spectroscopy as $\gamma=1 /\left(\pi c T_{2}\right)$. In the absence of inhomogeneous broadening, the experimental linewidth contains the contributions of population decay and the pure dephasing process, and the total width is related to the population relaxation time $T_{1}$ and the pure dephasing time $T_{2}^{\prime}$ as $\pi c \gamma=1 / T_{2}=1 /\left(2 T_{1}\right)+1 / T_{2}^{\prime}$ [9-12]. The most significant difference between these two relaxation mechanisms is in their temperature dependence; the pure dephasing relaxation shows steeper temperature dependence than the population relaxation at low temperatures. Therefore, the temperature behavior of linewidths or the relaxation times can be used to identify the dominant mechanisms involved in the overall relaxation.

In general, measurement of the homogeneous linewidth of impurity molecules in condensed phases by frequency domain spectroscopy is very difficult, because perturbations from the condensed phases that cause inhomogeneous broadening can hardly be avoided. Thus, relaxation times have been observed using special techniques of time domain spectroscopy such as photon echo, etc. [3,4,13,14]. However, since the time domain spectroscopy always suffers from low frequency resolution, observation of the dephasing using high-resolution frequency domain spectroscopy is requisite for a detailed discussion on quantum level dependences of the relaxation, etc.

Recently, it has been shown that molecules embedded in parahydrogen crystals show extremely sharp vibrational absorption spectra $[15,16]$. Because of the quantum nature of solid hydrogen [17] and the spherical nature of the ground ( $v=0, J=0)$ parahydrogen molecule, the crystal provides a soft environment for a guest molecule, reducing the incidence of inhomogeneities. The spectral linewidth of vibrational transitions is sharp enough to resolve fine splittings due to crystal field interactions as well as quantized rotational motions.

In a series of papers [18-20], we have observed and analyzed the rotation-vibration transitions of methane molecules in parahydrogen crystals. We found that the rotational constants of methane in these crystals are only about 10\% smaller than those in the gas phase. Moreover, the Coriolis coupling constants are almost exactly the same as in the gas phase. The observed sharp and well-resolved spectra are apt for a detailed study of the vibrational relaxation by frequency domain spectroscopy, which is the scope of this paper. Here, we report a precise study on the temperature dependence of the spectrum of $\mathrm{CD}_{4}$ in order to identify the relaxation process of impurity molecules in the quantum crystal.

The method of crystal growth of solid hydrogen containing a small amount of methane has been described in previous papers $[15,18,20]$. Briefly, we made crystals in an enclosed copper cell which was attached to the base of a Dewar-type liquid He cryostat equipped with a temperature controller stage. Pure parahydrogen gas (ortho concentration $\leq 0.05 \%$ ) containing about $10 \mathrm{ppm}$ of methane was introduced continuously into the cell and kept at $8.5 \mathrm{~K}$ to grow almost transparent crystals. The degenerate stretching $\left(\nu_{3}\right)$ and bending $\left(\nu_{4}\right)$ vibrational transitions of $\mathrm{CD}_{4}$ were observed by a Fourier transform infrared (FTIR) spectrometer (Bruker, IFS-120HR). The resolution of the spectrometer was set to 0.01 or $0.0035 \mathrm{~cm}^{-1}$ depending on the linewidth. A higher resolution of $0.0035 \mathrm{~cm}^{-1}$ was employed when the FWHM was narrower than $0.1 \mathrm{~cm}^{-1}$ to avoid unwanted deformation of the spectral shape from the instrumental resolution. The Blackman-Harris three-term apodization function was used for the Fourier transformation of interferograms which resulted in a less deformed spectral line 
shape. An infrared optical bandpass filter along with low and high pass electric filters was used to make recordings with a better signal-to-noise ratio. The spectral line shape of both the $\nu_{3}$ and $\nu_{4}$ transitions was observed at temperatures between 3.7 and $8.5 \mathrm{~K}$. The temperature of the crystal was controlled by a heat controller (Scientific Instruments, Model 9650) with a stability of $\pm 0.01 \mathrm{~K}$ during the observation. Calibrated Si diode sensors were used to measure the temperature of the crystal. The liquid $\mathrm{He}$ of the cryostat was pumped by a rotary pump to achieve a temperature below $4.5 \mathrm{~K}$.

Figure 1 shows rovibrational spectra of the $\nu_{3}$ and $\nu_{4}$ transitions of $\mathrm{CD}_{4}$ in solid parahydrogen observed at three different temperatures. A low resolution spectrum observed at $4.5 \mathrm{~K}$ has been published in a previous paper [19], in which complete analysis of the spectrum was given. From Fig. 1, we see a drastic broadening of linewidths upon an increase in temperature. Moreover, it is seen that, at the same temperature, the width of the $\nu_{3}$ transition is always broader than that of the $\nu_{4}$ transition. The observed spectral change upon temperature was completely reversible between 3.7 and $8.5 \mathrm{~K}$. Above $8.5 \mathrm{~K}$, however, irreversible change of the spectral shape started to take place due to irreversible diffusional clustering of methane molecules in the parahydrogen crystal.

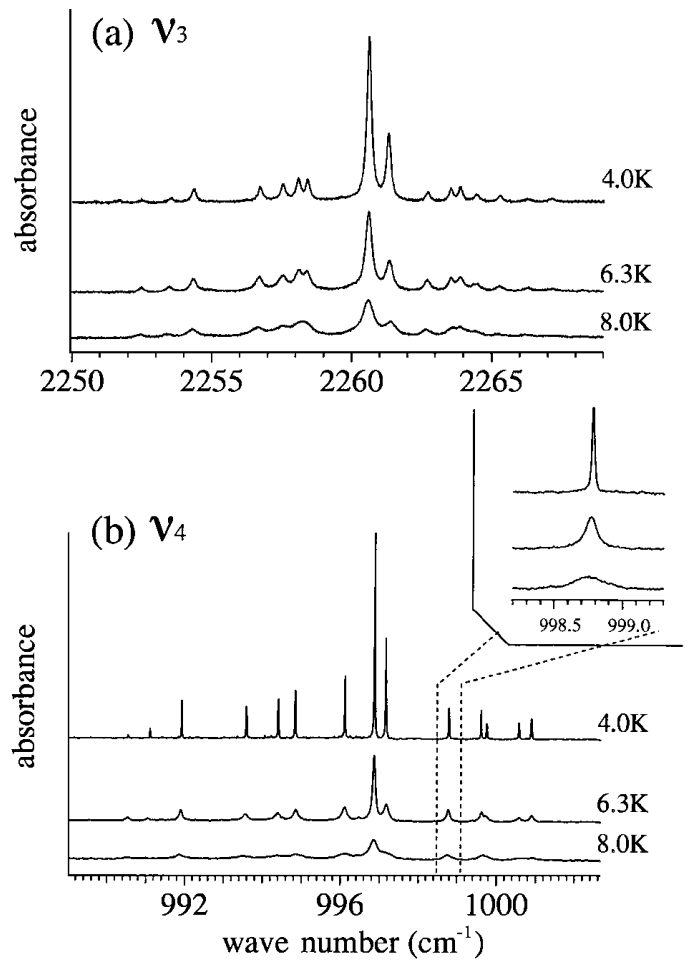

FIG. 1. Infrared absorption spectra of $\mathrm{CD}_{4}$ in the parahydrogen matrix observed at 4.0, 6.3, and 8.0 K. Trace (a) shows the $\nu_{3}$ triply degenerate stretching transition and trace (b) shows the $\nu_{4}$ triply degenerate bending transition. The spectra were taken with the resolution of $0.01 \mathrm{~cm}^{-1}$ at 6.3 and $8.0 \mathrm{~K}$ and of $0.0035 \mathrm{~cm}^{-1}$ at $4.0 \mathrm{~K}$. The inset in trace (b) shows the expansion of the peak at $998.8 \mathrm{~cm}^{-1}$.
Therefore, we limit our discussion here to the temperature dependence below $8.5 \mathrm{~K}$.

It was clearly noticed that all the observed absorption peaks have a Lorentzian line shape profile at all temperatures. Figure 2 compares a theoretical Lorentzian line shape (dotted curve) with the observed $\nu_{4}$ transition at $997.2 \mathrm{~cm}^{-1}$ (solid curve) as an example. The trace of difference drawn by the dashed curve in Fig. 2 clearly shows the observed line shape as a pure Lorentzian, which in turn indicates that the spectrum is completely free from inhomogeneous broadening. Absence of the inhomogeneous width must be due to the softness of the quantum crystal.

The FWHM temperature dependence for the lower frequency component of the $R(0)(J=1 \leftarrow 0, M= \pm 1 \leftarrow$ $0)$ transition is plotted in Fig. 3 for both the $\nu_{3}$ and $\nu_{4}$ transitions. The width increases monotonously with the increase in temperature. The linewidth of the $\nu_{4}$ band becomes $\sim 25$ times broader from 3.7 to $8.5 \mathrm{~K}$, while that of the $\nu_{3}$ band becomes only about 2.5 times broader. At $8.5 \mathrm{~K}$, the widths of the $\nu_{3}$ and $\nu_{4}$ transitions become equal.

Since the Debye temperature of solid hydrogen is known to be about $100 \mathrm{~K}\left(\simeq 70 \mathrm{~cm}^{-1}\right)$ [21], the width due to the population relaxation is expected to be approximately constant below $8.5 \mathrm{~K}$. Therefore the steep dependence that appeared in Fig. 3 must be attributed to the pure dephasing relaxation.

A number of theoretical studies on the temperature dependence of the pure dephasing width $1 /\left(\pi c T_{2}^{\prime}\right)$ have been reported $[7,8]$. McCumber and Sturge were the first who derived $T^{7}$ dependence of the linewidth for pure dephasing using the Debye-type acoustic phonon [22]. Krivoglaz derived equations for pure dephasing by a pseudolocal phonon and obtained the function of an Arrhenius-type $e^{-h \nu_{0} / k T}$ temperature dependence of the width at low temperatures where $\nu_{0}$ is the frequency of the local mode [23]. These theories were more generalized by Osad'ko [7] and Skinner and Hsu [8] independently using the nonperturbative approach.

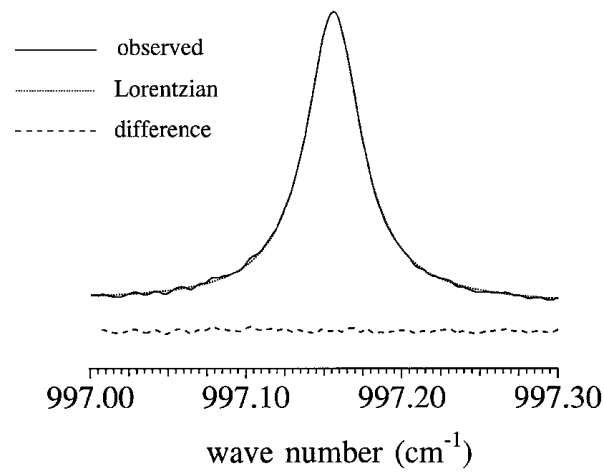

FIG. 2. Spectral profile of the $R(0)(J=1 \leftarrow 0, M=0 \leftarrow$ $0)$ transition of the $\nu_{4}$ band of $\mathrm{CD}_{4}$. Solid curve: Observed spectrum at $4.7 \mathrm{~K}$. Dotted curve: Theoretical Lorentzian line shape. Dashed curve: Fit residuals. 


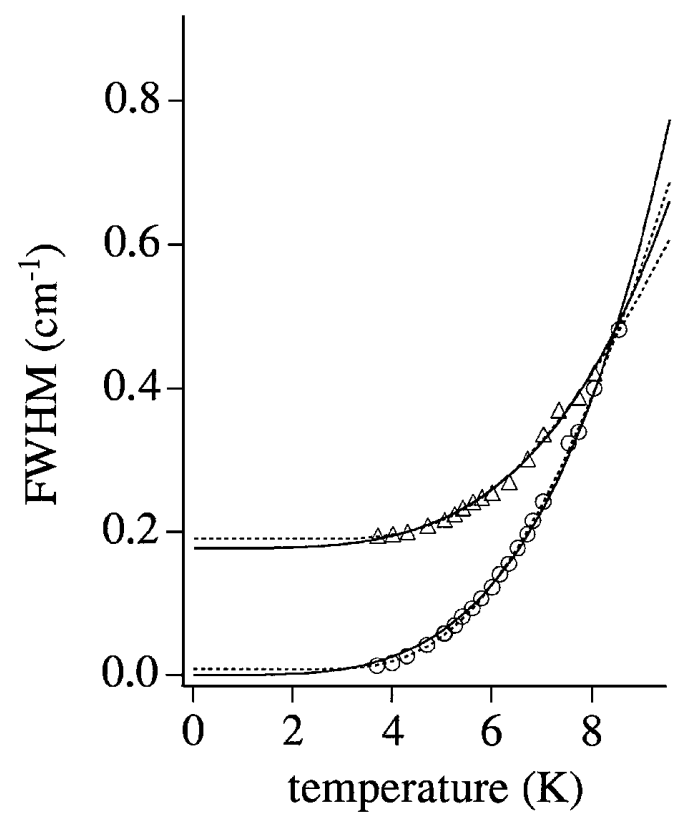

FIG. 3. Temperature dependence of the linewidth of the $R(0)$ $(J=1 \leftarrow 0, M= \pm 1 \leftarrow 0)$ transition of the $\nu_{3}(\triangle)$ and $\nu_{4}(\bigcirc)$ bands of $\mathrm{CD}_{4}$. The solid line shows the theoretical curve fitted by a function of $a+b T^{c}$. The optimized functional values are $a=0.177 \pm 0.006, b=(8.9 \pm 5.1) \times 10^{-5}$, and $c=3.82 \pm$ 0.27 for the $\nu_{3}$ transition and $a=0.0$ (fixed), $b=(1.13 \pm$ $0.15) \times 10^{-4}$, and $c=3.92 \pm 0.07$ for the $\nu_{4}$ transition [24]. The dotted line shows the theoretical curve of a type of $\alpha+$ $\beta e^{-h c \gamma / k T}$, where $h, c$, and $k$ represent the Planck constant, the speed of light, and the Boltzmann constant, respectively. The best fitting parameters are $\alpha=0.191 \pm 0.004, \beta=8.92 \pm$ 1.70 , and $\gamma=20.2 \pm 1.1 \mathrm{~cm}^{-1}$ for the $\nu_{3}$ transition and $\alpha=$ $0.008 \pm 0.002, \beta=13.46 \pm 0.67$, and $\gamma=19.7 \pm 0.3 \mathrm{~cm}^{-1}$ for the $\nu_{4}$ transition.

The observed temperature dependence was fitted by the least-squares method with both types of function of the power of $T$ and of the Arrhenius type. The best fitting functions are given in Fig. 3. If the pure dephasing is assumed to be caused by the low frequency acoustic phonon, a dependence of approximately $T^{4}$ was obtained for both the $\nu_{3}$ and $\nu_{4}$ transitions. On the other hand, the local mode frequency of about $20 \mathrm{~cm}^{-1}$ was obtained based on the dephasing model of local mode. Unfortunately both types of function fit quite well and it is hardly distinguishable which type of function is appropriate to describe the present dependence. Experimental data at lower temperatures are requisite to identify the mechanism. Subsequent observation of the width in the range of 1.0-4.0 K will be attempted. Nevertheless, it seems reasonable to suppose that the model of pure dephasing by the acoustic phonon is more appropriate since we could not find any data which supported the existence of the local phonon mode. Therefore, the $\sim T^{4}$ dependence obtained in this experiment is noteworthy. The discrepancy between the theory $\left(\sim T^{7}\right)$ and the experiment $\left(\sim T^{4}\right)$ must be ascribed to the quantum nature of the solid parahydrogen where lattice motions occur far from the harmonic oscillator [17].

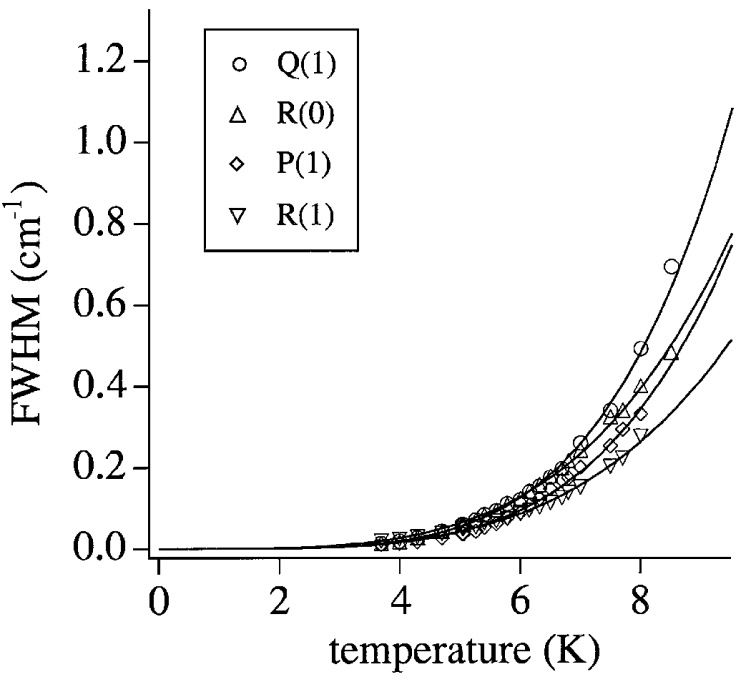

FIG. 4. Rotational level dependences on the linewidth of the $\nu_{4}$ transition. $Q(1)(\bigcirc)$ : transition of $J=1 \leftarrow 1, M=0 \leftarrow$ \pm 1 at $994.9 \mathrm{~cm}^{-1}$. $R(0)(\triangle)$ : transition of $J=1 \leftarrow 0, M=$ $0 \leftarrow 0$ at $997.2 \mathrm{~cm}^{-1}$. $P(1)(\diamond)$ : transition of $J=0 \leftarrow 1$, $M=0 \leftarrow \pm 1$ at $991.9 \mathrm{~cm}^{-1}$. $R(1)(\nabla)$ : transition of $J=$ $2 \leftarrow 1, M= \pm 1 \leftarrow 0$ at $998.8 \mathrm{~cm}^{-1}$. The solid lines show the theoretical curves fitted by a function of $b T^{c}$.

It should also be added that the dephasing rate depends not only on the vibrational quantum number but also on the rotational quantum numbers, $J$ and $M$. Some examples are given in Fig. 4. The different relaxation rates for different rotational levels indicate that intra- or intermolecular rotation-rotation interaction or rotation-phonon interaction is important in the dephasing process. Such clear evidence of the rotational quantum level dependence on dephasing has never been reported previously.

Turning now to the problem of the difference in the linewidths between the $\nu_{3}$ and $\nu_{4}$ transitions at the same temperatures, as is clearly seen in Fig. 3, the origin of the difference is attributed to the width extrapolated to $0 \mathrm{~K}$. With the assumption of pure dephasing by the acoustic phonon, the width of the $\nu_{3}$ band at $0 \mathrm{~K}$ is extrapolated to be $0.177 \mathrm{~cm}^{-1}$ while that of the $\nu_{4}$ is $0.000 \mathrm{~cm}^{-1}$ [24]. Since the pure dephasing width should vanish at the limit of $0 \mathrm{~K}$, the residual width is accounted for by the population relaxation, $T_{1}$. In the gas phase, the lifetime of the $\nu_{3}$ vibrational excited state has been observed to be much shorter than that of the $\nu_{4}$ state [25-28]. The rapid decay of the $\nu_{3}$ excited state in the gas phase is mainly attributed to the vibration-vibration energy transfer process. As for the interaction in solid parahydrogen, not only the intramolecular V-V transfer but also the intermolecular energy transfer due to the coupling between the stretching vibration of methane and lattice phonon may play an important role for the large population relaxation width of the $\nu_{3}$ transition. This needs further theoretical consideration.

In conclusion, we have investigated the vibrational dephasing of $\mathrm{CD}_{4}$ molecules in parahydrogen crystals by frequency domain spectroscopy. We found three important 
results concerning the dephasing: (1) $\sim T^{4}$ dependence of the pure dephasing width, (2) distinct dependence on the rotational quantum numbers $J$ and $M$, and (3) rapid $T_{1}$ relaxation of the $\nu_{3}$ transition. Since parahydrogen crystals can be characterized much better than any other molecular crystals, the present results will play a role for a deeper understanding of dephasing dynamics and the properties of quantum crystals. More precise experiments are presently underway.

The work described herein was supported in part by the Grant-in-Aid for Scientific Research of the Ministry of Education, Science, Culture and Sports of Japan. H. K. acknowledges the support from JSPS Research Fellowships for Young Scientists.

*Email address: momose@kuchem.kyoto-u.ac.jp

[1] L. E. Brus and V.E. Bondybey, J. Chem. Phys. 63, 786 (1975); V.E. Bondybey, J. Chem. Phys. 65, 5138 (1976).

[2] J. M. Wiesenfeld and C. B. Moore, J. Chem. Phys. 70, 930 (1979); L. Young and C.B. Moore, J. Chem. Phys. 81, 3137 (1984).

[3] H. Graener, T. Q. Ye, and A. Laubereau, Phys. Rev. B 41, 2597 (1990); H. Graener, G. Seifert, and A. Laubereau, Phys. Rev. Lett. 66, 2092 (1991).

[4] D. Zimdars, A. Tokmakoff, S. Chen, S. R. Greenfield, M. D. Fayer, T. I. Smith, and H. A. Schwettman, Phys. Rev. Lett. 70, 2718 (1993).

[5] M. Bonn, M. J. P. Brugmans, A. W. Kleyn, R. A. van Santen, and H. J. Bakker, Phys. Rev. Lett. 76, 2440 (1996); J. Chem. Phys. 105, 3431 (1996).

[6] C. E. Mungan, R. Lai, and A. J. Sievers, Chem. Phys. Lett. 270, 309 (1997).

[7] I. S. Osad'ko, Sov. Phys. Solid State 17, 2098 (1976); I. S. Osad'ko, Phys. Rep. 206, 43 (1991).

[8] J. L. Skinner and D. Hsu, Adv. Chem. Phys. 65, 1 (1986); J. L. Skinner and D. Hsu, J. Phys. Chem. 90, 4931 (1986); J. L. Skinner and D. Hsu, Chem. Phys. 128, 35 (1988).

[9] F. Bloch, Phys. Rev. 70, 460 (1946).

[10] A. Abragam, Principles of Nuclear Magnetism (Oxford University, London, 1961).

[11] L. Allen and J.H. Eberly, Optical Resonance and TwoLevel Atoms (Dover Publications, New York, 1975).
[12] K. Duppen, L. W. Molenkamp, J. B. W. Morsink, D. A. Wiersma, and H.P. Trommsdorff, Chem. Phys. Lett. 84, 421 (1981).

[13] W. H. Hesselink and D. A. Wiersma, J. Chem. Phys. 73, 648 (1980); L. W. Molenkamp and D. A. Wiersma, J. Chem. Phys. 80, 3054 (1984).

[14] S. Voelker, R. M. Macfarlane, A. Z. Genack, H. P. Trommsdorff, and J.H. van der Waals, J. Chem. Phys. 67, 1759 (1977); S. Voelker, R. M. Macfarlane, and J. H. van der Waals, Chem. Phys. Lett. 53, 8 (1978).

[15] T. Momose and T. Shida, Bull. Chem. Soc. Jpn. 71, 1 (1998).

[16] M.E. Fajardo and S. Tam, J. Chem. Phys. 108, 4237 (1998); S. Tam and M.E. Fajardo, Rev. Sci. Instrum. 70, 1926 (1999).

[17] J. Van Kranendonk, Solid Hydrogen, Theory of the Properties of Solid $\mathrm{H}_{2}, \mathrm{HD}$, and $\mathrm{D}_{2}$ (Plenum, New York, 1983).

[18] T. Momose, J. Chem. Phys. 107, 7695 (1997); T. Momose, M. Miki, T. Wakabayashi, T. Shida, M. C. Chan, S. S. Lee, and T. Oka, J. Chem. Phys. 107, 7707 (1997).

[19] H. Hoshina, T. Wakabayashi, T. Momose, and T. Shida, J. Chem. Phys. 110, 5728 (1999).

[20] S. Tam, M. E. Fajardo, H. Katsuki, H. Hoshina, T. Wakabayashi, and T. Momose, J. Chem. Phys. 111, 4191 (1999).

[21] P.C. Souers, Hydrogen Properties for Fusion Energy (University of California Press, Berkeley, 1986).

[22] D. E. McCumber and M. D. Sturge, J. Appl. Phys. 34, 1682 (1963).

[23] M. A. Krivoglaz, Sov. Phys. Solid State 6, 1340 (1964).

[24] During the fitting procedure, we fixed the first parameter $a$ of the function $a+b T^{c}$ equal to zero for the $\nu_{4}$ transition. Without the restriction, we obtained a fitting function of $-0.021+2.6 \times 10^{-4} T^{3.53}$. However, since the negative width at $0 \mathrm{~K}$ of $-0.021 \mathrm{~cm}^{-1}$ does not have any physical sense, we employ the restricted values as the best fitting parameters in the caption of Fig. 3.

[25] J. T. Yardley and C. B. Moore, J. Chem. Phys. 49, 1111 (1968); P. Hess, A. H. Kung, and C. B. Moore, J. Chem. Phys. 72, 5525 (1980).

[26] P. Varanasi, J. Quant. Spectrosc. Radiat. Transfer 11, 1711 (1971); P. Varanasi and G. D. T. Tejwani, J. Quant. Spectrosc. Radiat. Transfer 12, 849 (1971).

[27] E. Avramides and T.F. Hunter, Chem. Phys. 57, 441 (1981).

[28] D. Romanini and K. K. Lehmann, J. Mol. Spectrosc. 151, 54 (1992). 